\title{
Orthogonal polynomials and special functions
}

\author{
Elsa Graneland
}

Examensarbete i matematik, $15 \mathrm{hp}$ Handledare: Jörgen Östensson Examinator: Veronica Crispin Quinonez Juni 2020 



\section{Abstract}

This thesis is a brief introduction to the theory of orthogonal polynomials which is based on the theory of inner product spaces. We will also consider some special functions, especially the Bessel function.

We present definitions of orthonormal and monic orthogonal polynomials, and discuss the three-term recurrence relation, the Jacobi matrix and also a result concerning the zeros of the orthogonal polynomials.

Furthermore the Sturm-Liouville problem is presented and in particular, the Bessel function. Many polynomials and special functions are solutions to differential equations describing phenomena in nature. Lastly some applications to physics, e.g. quantum mechanics, are being presented. 


\section{Contents}

1 Introduction $\quad 4$

2 Inner product and inner product space $\quad 4$

3 Gram-Schmidt process $\quad 6$

4 Orthogonal polynomials $\quad 8$

4.1 Orthonormal and monic orthogonal polynomials . . . . . . . . . . 8

4.2 Three-term recurrence relation . . . . . . . . . . . . . . . 10

4.3 The Jacobi matrix . . . . . . . . . . . . . . . . . . . . . . . 12

4.4 Zeros of orthogonal polynomials . . . . . . . . . . . . . 13

5 Classical orthogonal polynomials $\quad 16$

5.1 Legendre polynomials . . . . . . . . . . . . . . . . . 16

5.2 Hermite polynomials . . . . . . . . . . . . . . . . 18

5.3 Laguerre polynomials . . . . . . . . . . . . . . . . . 20

6 Sturm-Liouville problems $\quad 21$

7 Applications $\quad 24$

7.1 Eigenvalues of the Laplace operator . . . . . . . . . . . . . . . . . . . 24

7.1.1 Eigenvalue problem in a disk . . . . . . . . . . . 25

7.1.2 Eigenvalue problem in a ball . . . . . . . . . . . . . . 26

7.2 Schrödinger equation . . . . . . . . . . . . . . . . 28

7.2 .1 Harmonic oscillator . . . . . . . . . . . . . . . . . . . . . . 29

7.2 .2 Hydrogen atom . . . . . . . . . . . . . . . 30

8 References $\quad 33$ 


\section{Introduction}

The theory of orthogonal polynomials and special functions is of intrinsic interest to many parts of mathematics. Moreover, it can be used to explain many physical and chemical phenomena. For example, the vibrations of a drum head can be explained in terms of special functions known as Bessel functions. And the solutions of the Schrödinger equation for a harmonic oscillator can be described using orthogonal polynomials known as Hermite polynomials. Furthermore, the eigenfunctions for the Schrödinger operator associated with the hydrogen atom are described in terms orthogonal polynomials known as Laguerre polynomials.

In Section 2 the definitions of inner product and inner product space are presented, and in Section 3 the Gram-Schmidt algorithm is described. In Section 4 the orthonormal and monic orthogonal polynomials are defined. This is followed by a discussion about the three-term recurrence relation, the Jacobi matrix and the zeros of orthogonal polynomials. This thesis considers three of the classical orthogonal polynomials, which are presented in Section 5. In Section 6 we discuss Sturm-Liouville problems. The last section in this thesis, Section 7, present the applications mentioned above.

\section{Inner product and inner product space}

The standard scalar products of $\mathbb{R}^{n}$ and $\mathbb{C}^{n}$ satisfy some calculation rules. These are taken as axioms in a general inner product space.

Definition 2.1. The inner product $\langle\cdot, \cdot\rangle$ on a vector space $X$ is a mapping of $X \times X$ in to the scalar field $K(=\mathbb{R}$ or $\mathbb{C})$ satisfying the following. For all vectors $x, y$ and $z$ and scalars $\alpha$ we have:

1. $\langle x+y, z\rangle=\langle x, z\rangle+\langle y, z\rangle$.

2. $\langle\alpha x, y\rangle=\alpha\langle x, y\rangle$.

3. $\langle x, y\rangle=\overline{\langle y, x\rangle}$.

4. $\langle x, x\rangle \geq 0$ and equal if and only if $x=0$.

The inner product on $X$ defines a norm on $X$ given by

$$
\|x\|=\sqrt{\langle x, x\rangle}
$$

and a distance function, or metric, on $X$ given by

$$
d(x, y)=\|x-y\|=\sqrt{\langle x-y, x-y\rangle} .
$$

When an inner product space is complete, the space is called a Hilbert space and is usually denoted by $H$.

Remark. It follows from the definition above, that

$$
\begin{aligned}
& \langle\alpha x+\beta y, z\rangle=\alpha\langle x, z\rangle+\beta\langle y, z\rangle . \\
& \langle x, \alpha y+\beta z\rangle=\bar{\alpha}\langle x, y\rangle+\bar{\beta}\langle x, z\rangle .
\end{aligned}
$$

Due to the conjugate in the second variable, one says that the inner product is sesquilinear, meaning " $1 \frac{1}{2}$ times linear". 
Definition 2.2. Two elements $x$ and $y$ in an inner product space $X$ are said to be orthogonal if $\langle x, y\rangle=0$. A set of vectors is called a orthonormal set if these vectors are pairwise orthogonal and of norm 1.

\section{Example 1. Euclidean space $\mathbb{R}^{n}$.}

Given vectors $x=\left(x_{1}, x_{2}, \ldots, x_{n}\right)$ and $y=\left(y_{1}, y_{2}, \ldots, y_{n}\right)$ in $\mathbb{R}^{n}$ an inner product is defined by

$$
\langle x, y\rangle=x_{1} y_{1}+x_{2} y_{2}+\ldots+x_{n} y_{n}=\sum_{j=1}^{n} x_{j} y_{j} .
$$

This makes $\mathbb{R}^{n}$ into a Hilbert space.

\section{Example 2. Unitary space $\mathbb{C}^{n}$.}

The standard inner product on $\mathbb{C}^{n}$ is given by

$$
\langle x, y\rangle=\sum_{j=1}^{n} x_{j} \overline{y_{j}}
$$

This makes $\mathbb{C}^{n}$ into a Hilbert space.

Example 3. The space $C[a, b]$.

Let $C[a, b]$ denote the space complex-valued continuous functions defined on the interval $[a, b]$. An inner product on $C[a, b]$ is given by

$$
\langle f, g\rangle=\int_{a}^{b} f(x) \overline{g(x)} d x .
$$

This space is not complete in the metric induced by the scalar product.

Example 4. The space $L^{2}(\mathbb{R}, d \mu)$.

Given a positive Borel measure $d \mu$ on $\mathbb{R}$, we let $L^{2}(\mathbb{R}, d \mu)$ denote the equivalent classes of all Borel measurable functions such that

$$
\int_{\mathbb{R}}|f|^{2} d \mu<\infty
$$

Two functions are considered equivalent if they agree $\mu$-almost everywhere. An inner product on $L^{2}[\mathbb{R}, d \mu]$ is given by

$$
\langle f, g\rangle=\int_{\mathbb{R}} f(x) \overline{g(x)} d \mu .
$$

This makes $L^{2}(\mathbb{R}, d \mu)$ into a Hilbert space.

For the classical orthogonal polynomials it holds that

$$
d \mu=\omega(x) d x .
$$

Moreover the so-called $\omega$ usually vanishes outside some interval $[a, b]$. In this case we write $L^{2}([a, b], \omega(x) d x)$, and the scalar product is given by

$$
\langle f, g\rangle=\int_{a}^{b} f(x) \overline{g(x)} \omega(x) d x .
$$

In case $\langle f, g\rangle=0$ we say that $f$ and $g$ are orthogonal with respect to the weight $\omega$ on $[a, b]$.

For more about the theory of inner product spaces and Hilbert spaces, see [3]. 


\section{Gram-Schmidt process}

The Gram Schmidt process is an algorithm that is used to turn any linearly independent set of vectors into a orthonormal set of vectors.

We denote the linearly independent set of vectors by $x_{j}$, and the resulting orthonormal set of vectors by $e_{j}$. The steps of the algorithm are as follows:

- First step: The first element of the orthonormal sequence, $e_{1}$, will be obtained from

$$
e_{1}=\frac{1}{\left\|x_{1}\right\|} x_{1}
$$

- Second step: All the following steps will include two parts: first create a vector orthogonal to the previous vector(s), then normalize it. We create $v_{2}$ as

$$
v_{2}=x_{2}-\left\langle x_{2}, e_{1}\right\rangle e_{1},
$$

and then normalize it:

$$
e_{2}=\frac{1}{\left\|v_{2}\right\|} v_{2}
$$

- Third step: We create $v_{3}$ as

$$
v_{3}=x_{3}-\left\langle x_{3}, e_{1}\right\rangle e_{1}-\left\langle x_{3}, e_{2}\right\rangle e_{2}
$$

and then normalize:

$$
e_{3}=\frac{1}{\left\|v_{3}\right\|} v_{3}
$$

The algorithm proceeds by induction.

- nth step: Suppose that $\left\{e_{1}, \ldots, e_{n-1}\right\}$ is an orthonormal set such that $\operatorname{span}\left\{e_{1}, \ldots, e_{n-1}\right\}=\operatorname{span}\left\{x_{1}, \ldots, x_{n-1}\right\}$. The vector $v_{n}$ is defined by:

$$
v_{n}=x_{n}-\sum_{k=1}^{n-1}\left\langle x_{n}, e_{k}\right\rangle e_{k} .
$$

Note that $v_{n}$ is a non-zero vector. Otherwise $x_{n}$ would belong to the span of $\left\{x_{1}, \ldots, x_{n-1}\right\}$ and the set $\left\{x_{1}, \ldots, x_{n}\right\}$ would be linearly dependent. Note also that $v_{n}$ is orthogonal to all the vectors $e_{1}, \ldots, e_{n-1}$.

Normalizing $v_{n}$ :

$$
e_{n}=\frac{1}{\left\|v_{n}\right\|} v_{n}
$$

we therefore obtain an orthonormal set $\left\{e_{1}, \ldots, e_{n}\right\}$ with $\operatorname{span}\left\{e_{1}, \ldots, e_{n}\right\}=$ $\operatorname{span}\left\{x_{1}, \ldots, x_{n}\right\}$.

\section{Example 5. Gram-Schmidt procedure on vectors in $\mathbb{R}^{3}$}

Consider the two vectors $x_{1}=(1,1,0)$ and $x_{2}=(1,2,1)$. The Gram-Schmidt procedure can be used to obtain a set $\left\{e_{1}, e_{2}\right\}$ that is orthonormal with respect to the standard scalar product in $\mathbb{R}^{3}$. 
- First step: The vector $e_{1}$ is obtained by normalizing $x_{1}$ :

$$
e_{1}=\frac{1}{\left\|x_{1}\right\|} x_{1}=\frac{1}{\sqrt{2}}(1,1,0) \text {. }
$$

- Second step: We create $v_{2}$ as:

$$
v_{2}=x_{2}-\left\langle x_{2}, e_{1}\right\rangle e_{1}=(1,2,1)-\frac{3}{2}(1,1,0)=\frac{1}{2}(-1,1,2) .
$$

And now we normalize:

$$
e_{2}=\frac{1}{\left\|v_{2}\right\|} v_{2}=\frac{1}{\sqrt{6}}(-1,1,2)
$$

\section{Example 6. Gram-Schmidt process on polynomials}

Consider the set $u=\left\{1, x, x^{2}\right\}$, and let $u_{1}=1, u_{2}=x$ and $u_{3}=x^{2}$. The Gram-Schmidt process can be used to obtain a set $\left\{e_{1}, e_{2}, e_{3}\right\}$ that is orthonormal with respect to the inner product

$$
\langle f, g\rangle=\int_{-1}^{1} f(x) g(x) d x .
$$

- First step: The first element of the orthonormal sequence, $e_{1}$, will be obtained from

$$
e_{1}=\frac{1}{\left\|u_{1}\right\|} u_{1}=\frac{1}{\sqrt{2}}
$$

- Second step: We create $v_{2}$ as:

$$
v_{2}=u_{2}-\left\langle u_{2}, e_{1}\right\rangle e_{1}=x-\langle x, 1\rangle=x-\int_{-1}^{1} x \cdot 1 d x=x .
$$

And then make it normalized:

$$
e_{2}=\frac{1}{\left\|v_{2}\right\|} v_{2}=\frac{x}{\sqrt{\frac{2}{3}}}=\sqrt{\frac{3}{2}} x .
$$

- Third step: We create $v_{3}$ as:

$$
\begin{aligned}
v_{3} & =u_{3}-\left\langle u_{3}, e_{1}\right\rangle e_{1}-\left\langle u_{3}, e_{2}\right\rangle e_{2} \\
& =x^{2}-\left(\int_{-1}^{1} x^{2} \cdot \frac{1}{\sqrt{2}} d x\right) \cdot \frac{1}{\sqrt{2}}-\left(\int_{-1}^{1} x^{2} \cdot \sqrt{\frac{3}{2}} x d x\right) \cdot \sqrt{\frac{3}{2}} x \\
& =x^{2}-\frac{1}{2} \cdot \frac{2}{3}-0=x^{2}-\frac{1}{3}
\end{aligned}
$$

Note that

$$
\left\|v_{3}\right\|^{2}=\int_{-1}^{1}\left(x^{2}-\frac{1}{3}\right)^{2} d x=\frac{8}{45}
$$

and therefore:

$$
e_{3}=\frac{1}{\left\|v_{3}\right\|} v_{3}=\frac{3 \sqrt{5}}{2 \sqrt{2}}\left(x^{2}-\frac{1}{3}\right)
$$

These are, up to a multiplicative factor, the first three so-called Legendre polynomials. 


\section{Orthogonal polynomials}

\subsection{Orthonormal and monic orthogonal polynomials}

Let $d \mu$ be a positive Borel measure on $\mathbb{R}$ having finite moments, that is

$$
\int_{\mathbb{R}}|x|^{m} d \mu<\infty
$$

for all integers $m \geq 0$. Furthermore assume that supp $\mu$ contains infinitely many points, i.e. $\#(\operatorname{supp} \mu)=\infty$. Then the set $\left\{1, x, x^{2}, \ldots\right\}$ is linearly independent. The orthonormal polynomials, $p_{n}(x)$, satisfy

$$
\int p_{n}(x) p_{m}(x) d \mu=\delta_{n m}
$$

where

$$
\delta_{n m}= \begin{cases}0, & n \neq m, \\ 1, & n=m .\end{cases}
$$

We assume that the coefficient of the highest order term is positive:

$$
p_{n}(x)=\gamma_{n} x^{n}+\ldots, \quad \gamma_{n}>0 .
$$

This makes the $p_{n}$ 's unique. They can be obtained by applying the Gram-Schmidt process to the set $\left\{1, x, x^{2}, \ldots\right\}$.

The orthogonal polynomials having leading coefficient 1 are called monic orthogonal polynomials. We denote them by $\pi_{n}(x)$, i.e. $\pi_{n}(x)=p_{n}(x) / \gamma_{n}$.

Let $M=M_{n}$ be the $(n+1) \times(n+1)$ matrix where all the elements are

$$
M=\left(M_{i j}\right)=\left(\int x^{i+j} d \mu(x)\right)_{0 \leq i, j \leq n},
$$

and let $D_{n}$ denote its determinant:

$$
D_{n}=\operatorname{det} M=\left|\begin{array}{cccc}
\int s^{0} d \mu & \int s d \mu & \cdots & \int s^{n} d \mu \\
\int s d \mu & \int s^{2} d \mu & \cdots & \int s^{n+1} d \mu \\
\vdots & \vdots & \vdots & \vdots \\
\int s^{n-1} d \mu & \int s^{n} d \mu & \cdots & \int s^{2 n-1} d \mu \\
\int s^{n} d \mu & \int s^{n+1} d \mu & \cdots & \int s^{2 n} d \mu
\end{array}\right| .
$$

Note that

$$
\begin{aligned}
0 & \leq\left\|\sum_{i=0}^{n} t_{i} x^{i}\right\|_{L^{2}(\mathbb{R}, d \mu)}^{2}=\left\langle\sum_{i=0}^{n} t_{i} x^{i}, \sum_{j=0}^{n} t_{j} x^{j}\right\rangle_{L^{2}(\mathbb{R}, d \mu)} \\
& =\sum_{i, j=0}^{n} t_{i} t_{j} \int_{\mathbb{R}} x^{i+j} d \mu=\sum_{i, j=0}^{n} t_{i} t_{j} M_{i, j}=\langle M t, t\rangle_{\mathbb{R}^{n+1}},
\end{aligned}
$$

where $t=\left\{t_{0}, \ldots, t_{n}\right\}^{T} \in \mathbb{R}^{n+1}$. Since $\#(\operatorname{supp} \mu)=\infty$ it follows that the matrix $M$ is strictly positive definite, and therefore

$$
D_{n}>0
$$


For notational convenience we set $D_{-1}=1$. Now set $D_{0}(x)=1$ and for $n \geq 1$

$$
D_{n}(x)=\left|\begin{array}{cccc}
\int s^{0} d \mu & \int s d \mu & \cdots & \int s^{n} d \mu \\
\int s d \mu & \int s^{2} d \mu & \cdots & \int s^{n+1} d \mu \\
\vdots & \vdots & \vdots & \vdots \\
\int s^{n-1} d \mu & \int s^{n} d \mu & \cdots & \int s^{2 n-1} d \mu \\
1 & x & \cdots & x^{n}
\end{array}\right|
$$

There is an explicit formula for the orthonormal polynomials, due to Heine.

Theorem 1. Let $p_{n}(x)$ for $n \geq 0$ be the sequence of polynomials orthogonal with respect to $d \mu$. Then

$$
p_{n}(x)=\frac{1}{\sqrt{D_{n-1} D_{n}}} D_{n}(x) .
$$

Proof. To prove that

$$
p_{n}(x)=\frac{1}{\sqrt{D_{n-1} D_{n}}} D_{n}(x)
$$

we write $\tilde{p}_{n}(x)=\frac{1}{\sqrt{D_{n-1} D_{n}}} D_{n}(x)$. For all $j<n$, multiplying with $x^{j}$ we get following expression

$$
\begin{gathered}
\sqrt{D_{n} D_{n-1}} \int x^{j} \tilde{p}_{n}(x) d \mu= \\
=\left|\begin{array}{cccc}
\int s^{0} d \mu & \int s d \mu & \cdots & \int s^{n} d \mu \\
\int s d \mu & \int s^{2} d \mu & \cdots & \int s^{n+1} d \mu \\
\vdots & \vdots & \vdots & \vdots \\
\int s^{n-1} d \mu & \int s^{n} d \mu & \cdots & \int s^{2 n-1} d \mu \\
\int x^{j} d \mu & \int x^{j+1} d \mu & \cdots & \int x^{j+n} d \mu
\end{array}\right| .
\end{gathered}
$$

Note that the last row in the matrix above is identical to some other row lying above. When two rows in a matrix are identical, the determinant will be 0 , as seen e.g. using row operations. That gives us

$$
\int x^{j} \tilde{p}_{n}(x) d \mu=0
$$

which also implies that $\tilde{p_{n}}(x) \perp \tilde{p_{m}}(x)$ for all $m<n$. Furthermore we want to show that $\left\|\tilde{p}_{n}\right\|^{2}=1$. Because of the equation $\tilde{p}_{n}(x)=\frac{1}{\sqrt{D_{n-1} D_{n}}} D_{n}(x)$, we have the following

$$
\left\|\tilde{p}_{n}\right\|^{2}=\left\langle\tilde{p}_{n}, \tilde{p}_{n}\right\rangle=\frac{1}{D_{n-1} D_{n}}\left\langle D_{n}(x), D_{n}(x)\right\rangle
$$

The right hand of this equation can be expressed using the definition of the inner product:

$$
\left\|\tilde{p}_{n}\right\|^{2}=\frac{1}{D_{n-1} D_{n}} \int D_{n}(x) D_{n}(x) d \mu .
$$


Expanding the second factor $D_{n}(x)$ along the last row, it follows that this can be rewritten as

$$
\left\|\tilde{p}_{n}\right\|^{2}=\frac{1}{D_{n-1} D_{n}} \int D_{n}(x)\left(D_{n-1} x^{n}+\cdots\right) d \mu
$$

where the dots represent the terms of lower order.

Note that all the terms of lower order will be orthogonal to $D_{n}(x)$ and therefore these integrals will be zero. What we have left is the following expression:

$$
\left\|\tilde{p}_{n}\right\|^{2}=\frac{1}{D_{n}} \int D_{n}(x) x^{n} d \mu .
$$

Using expansion along the last row one realizes that this can be rewritten as:

$$
\left\|\tilde{p}_{n}\right\|^{2}=\frac{1}{D_{n}}\left|\begin{array}{cccc}
\int x^{0} d \mu & \int x d \mu & \cdots & \int x^{n} d \mu \\
\int x d \mu & \int x^{2} d \mu & \cdots & \int x^{n+1} d \mu \\
\vdots & \vdots & \vdots & \vdots \\
\int x^{n-1} d \mu & \int x^{n} d \mu & \cdots & \int x^{2 n-1} d \mu \\
\int x^{n} d \mu & \int x^{n+1} d \mu & \cdots & \int x^{2 n} d \mu
\end{array}\right|,
$$

where the determinant is exactly the same thing as $D_{n}$. Thus

$$
\left\|\tilde{p}_{n}\right\|^{2}=\frac{1}{D_{n}} D_{n}=1
$$

Finally, expanding $D_{n}(x)$ along the last row one sees that the coefficient for the highest order term in $\tilde{p}_{n}$ is given by

$$
\frac{1}{\sqrt{D_{n-1} D_{n}}} \cdot D_{n-1}=\sqrt{\frac{D_{n-1}}{D_{n}}}>0 .
$$

Hence $\tilde{p}_{n}(x)=p_{n}(x)$.

\subsection{Three-term recurrence relation}

Since $x p_{k}(x)$ is a polynomial of degree $k+1$, it can be written as a sum

$$
x p_{k}(x)=\sum_{j=0}^{k+1} c_{j} p_{j}(x)
$$

for some constants $c_{j}$. Suppose that $k \geq 1$ and that $l<k-1$. Then, due to orthogonality, we have following expression:

$$
\int x p_{k}(x) p_{l}(x) d \mu=\int p_{k}(x) x p_{l}(x) d \mu=0,
$$


since $x p_{l}(x)$ is a polynomial of degree $l+1<k$. On the other hand, by (4.7) we have

$$
\int x p_{k}(x) p_{l}(x) d \mu=\sum_{j=0}^{k+1} c_{j} \int p_{j}(x) p_{l}(x) d \mu=c_{l} .
$$

By comparing equation (4.8) and equation (4.9), we see that for all $l<k-1$ it holds that $c_{l}=0$. That gives us only three terms left in (4.7): when $l=k-1, l=k$ and $l=k+1$. Therefore, the polynomial $x p_{k}(x)$ can be expressed as

$$
x p_{k}(x)=c_{k+1} p_{k+1}(x)+c_{k} p_{k}(x)+c_{k-1} p_{k-1}(x), \quad k \geq 1 .
$$

This is the famous three-term recurrence formula. Obviously, we also have

$$
x p_{0}(x)=c_{1} p_{1}(x)+c_{0} p_{0}(x), \quad k=0 .
$$

Set

$$
\begin{gathered}
a_{k}=\int x p_{k}^{2}(x) d \mu \\
b_{k}=\int x p_{k+1}(x) p_{k}(x) d \mu
\end{gathered}
$$

for $k \geq 0$. Multiplying equation (4.10) by $p_{k}(x)$ and integrating with respect to $d \mu$, we get:

$$
a_{k}=\int x p_{k}^{2}(x) d \mu=0+c_{k} \int p_{k}^{2}(x) d \mu+0=c_{k} .
$$

Similarly multiplying equation (4.10) by $p_{k+1}(x)$ and integrating with respect to $d \mu$, we get:

$$
b_{k}=\int x p_{k+1}(x) p_{k}(x) d \mu=c_{k+1} \int p_{k+1}^{2} d \mu+0+0=c_{k+1} .
$$

Finally, multiplying equation (4.10) by $p_{k-1}(x)$ and integrating with respect to $d \mu$, we get:

$$
b_{k-1}=\int x p_{k}(x) p_{k-1}(x) d \mu=0+0+c_{k-1} \int p_{k-1}^{2} d \mu=c_{k-1} .
$$

Doing similar calculation for the case $k=0$ we get:

$$
\begin{gathered}
x p_{k}(x)=b_{k} p_{k+1}(x)+a_{k} p_{k}(x)+b_{k-1} p_{k-1}(x), \quad k \geq 1, \\
x p_{0}(x)=b_{0} p_{1}(x)+a_{0} p_{0}(x), \quad k=0 .
\end{gathered}
$$

The leading coefficient of the left-hand side of the equation (4.15) is $\gamma_{k}$ and on the right-hand side $b_{k} \cdot \gamma_{k+1}$. Therefore:

$$
\gamma_{k}=b_{k} \cdot \gamma_{k+1}
$$


so that

$$
b_{k}=\frac{\gamma_{k}}{\gamma_{k+1}}>0
$$

If we set $T$ to be the tridiagonal matrix

$$
T=\left(\begin{array}{cccc}
a_{0} & b_{0} & 0 & \cdots \\
b_{0} & a_{1} & b_{1} & \ddots \\
0 & b_{1} & a_{2} & \ddots \\
\vdots & \ddots & \ddots & \ddots
\end{array}\right)
$$

the equations (4.15) and (4.16) can be rewritten as

$$
T\left(\begin{array}{c}
p_{0}(x) \\
p_{1}(x) \\
\vdots \\
p_{k}(x) \\
\vdots
\end{array}\right)=x\left(\begin{array}{c}
p_{0}(x) \\
p_{1}(x) \\
\vdots \\
p_{k}(x) \\
\vdots
\end{array}\right)
$$

\subsection{The Jacobi matrix}

Definition 4.1. The Jacobi matrix is defined as the symmetric matrix $T$ such that:

$$
T=\left(\begin{array}{ccccc}
a_{0} & b_{0} & 0 & \cdots & 0 \\
b_{0} & a_{1} & b_{1} & \ddots & \vdots \\
0 & b_{1} & a_{2} & \ddots & 0 \\
\vdots & \ddots & \ddots & \ddots & b_{n-2} \\
0 & \cdots & 0 & b_{n-2} & a_{n-1}
\end{array}\right), \quad b_{j}>0
$$

in the finite-dimensional case, and:

$$
T=\left(\begin{array}{cccc}
a_{0} & b_{0} & 0 & \cdots \\
b_{0} & a_{1} & b_{1} & \ddots \\
0 & b_{1} & a_{2} & \ddots \\
\vdots & \ddots & \ddots & \ddots
\end{array}\right), \quad b_{j}>0,
$$

in the infinite-dimensional case.

Theorem 2. Let $T$ be a finite $n \times n$ Jacobi matrix. Then the following holds:

1. The spectrum of $T$ is simple. Thus, there exist $n$ distinct eigenvalues $\lambda_{1}, \lambda_{2}, \ldots, \lambda_{n}$.

2. If $f=(f(1), f(2), \ldots, f(n))^{T} \neq 0$ is an eigenvector of $T$, i.e., $T f=\lambda f$, then $f(1) \neq 0$ and $f(n) \neq 0$.

Proof. We begin by proving the second statement. Assume that:

$$
(T-\lambda I) f=0
$$


where $f=(f(1), f(2), \ldots, f(n))^{T}$. The first row of equation (4.20) reads:

$$
\left(a_{0}-\lambda\right) f(1)+b_{0} f(2)=0 .
$$

For the proof we use proof by contradiction. Suppose that $f(1)=0$. Since $b_{0}>0$ it follows from equation $(4.21)$ that $f(2)=0$. The equations in the middle rows of $(4.20)$ can be written as

$$
b_{i-2} f(i-1)+\left(a_{i-1}-\lambda\right) f(i)+b_{i-1} f(i+1)=0
$$

for $2 \leq i<n$. From equation (4.22), with $i=2$, and get following expression:

$$
b_{0} f(1)+\left(a_{1}-\lambda\right) f(2)+b_{1} f(3)=0
$$

Since $f(1)=0, f(2)=0$ and $b_{1}>0$ it follows that $f(3)=0$, too. Continuing in this manner, setting $i=3, \ldots, i=n-1$, it follows $f(1)=f(2)=\ldots=f(n)=0$. This means that, if $f(1)=0$, then $f=0$. Therefore $f(1) \neq 0$. That $f(n) \neq 0$ follows from a similar reasoning, starting from the last row.

For the proof of the first statement, we suppose that $f$ and $\tilde{f}$ are two (non-zero) eigenvectors with the same eigenvalue $\lambda$. We can find a pair $(a, \tilde{a}) \neq(0,0)$ such that $g=a f+\tilde{a} \tilde{f}$ has $g(1)=0$. For example we may take $a=1$ and $\tilde{a}=-\frac{f(1)}{\tilde{f}(1)}$. Since $g$ is in the same eigenspace it follows, from the proof of the second statement, that $g=0$. This means that $f$ and $\tilde{f}$ are linearly dependent. So each eigenspace in one-dimensional. Since the matrix $T$ is symmetric, it follows that it must have $n$ distinct real eigenvalues.

\subsection{Zeros of orthogonal polynomials}

When considering the zeros of orthogonal polynomials, there are some properties that characterise the zeros. We will focus on two of them:

Theorem 3. Let $d \mu$ be a Borel measure with finite moments and infinite support, and let $p_{n}(x)$ be the associated orthonormal polynomials. Then,

1. The $n$ zeros of $p_{n}(x)$, are real and simple.

2. The zeros of $p_{n+1}(x)$ and $p_{n}(x)$ interlace, that is, between any two zeros of $p_{n+1}(x)$ lies exactly one zero of $p_{n}(x)$, and vice versa.

Proof of 1 . When proving the first property we may assume, without loss of generality, that $\int d \mu=1$. Let $T$ be the Jacobi matrix such that

$$
T=\left(\begin{array}{cccc}
a_{0} & b_{0} & 0 & \cdots \\
b_{0} & a_{1} & b_{1} & \ddots \\
0 & b_{1} & a_{2} & \ddots \\
\vdots & \ddots & \ddots & \ddots
\end{array}\right)
$$

We know that 
$0=(T-\lambda)\left(\begin{array}{c}p_{0}(\lambda) \\ p_{1}(\lambda) \\ \vdots\end{array}\right)=\left(\begin{array}{ccccccccc}a_{0}-\lambda & b_{0} & 0 & \cdots & & & & \\ b_{0} & a_{1}-\lambda & b_{1} & & & & \\ 0 & b_{1} & a_{2}-\lambda & \ddots & & & \\ \\ \cdots & & \ddots & \ddots & b_{n-2} & & \\ & & & b_{n-2} & a_{n-1}-\lambda & b_{n-1} & & \\ & & & & b_{n-1} & a_{n}-\lambda & b_{n} & \\ & & & & & & \ddots & \ddots\end{array}\right)\left(\begin{array}{c}p_{0}(\lambda) \\ p_{1}(\lambda) \\ \vdots\end{array}\right)$.

Hence, $p_{n}(\lambda)=0$ if and only if

$$
\left(p_{0}(\lambda), \ldots, p_{n-1}(\lambda)\right)=\left(1, p_{1}(\lambda), . ., p_{n-1}(\lambda)\right) \neq 0,
$$

is an eigenvector of the $n \times n$ Jacobi matrix

$$
\left(\begin{array}{ccccc}
a_{0} & b_{0} & 0 & \cdots & 0 \\
b_{0} & a_{1} & b_{1} & \ddots & \vdots \\
0 & b_{1} & a_{2} & \ddots & 0 \\
\vdots & \ddots & \ddots & \ddots & b_{n-2} \\
0 & \cdots & 0 & b_{n-2} & a_{n-1}
\end{array}\right)
$$

with eigenvalue $\lambda$. Therefore, the zeros of $p_{n}$ are the same as the eigenvalues of the matrix in equation (4.24). But, and due to Theorem 2 this matrix has $n$ real and simple zeros.

Proof of 2. The roots of $p_{n}(x)$ and $p_{n+1}(x)$ are eigenvalues of the two matrices $T_{n-1}$ and $T_{n}$, where

Consider

$$
\begin{gathered}
T_{n-1}=\left(\begin{array}{ccccc}
a_{0} & b_{0} & 0 & \cdots & 0 \\
b_{0} & a_{1} & b_{1} & \ddots & \vdots \\
0 & b_{1} & a_{2} & \ddots & 0 \\
\vdots & \ddots & \ddots & \ddots & b_{n-2} \\
0 & \cdots & 0 & b_{n-2} & a_{n-1}
\end{array}\right), \\
T_{n}=\left(\begin{array}{ccccc}
a_{0} & b_{0} & 0 & \cdots & 0 \\
b_{0} & a_{1} & b_{1} & \ddots & \vdots \\
0 & b_{1} & a_{2} & \ddots & 0 \\
\vdots & \ddots & \ddots & \ddots & b_{n-1} \\
0 & \cdots & 0 & b_{n-1} & a_{n}
\end{array}\right)
\end{gathered}
$$

$$
\left\langle e_{n},\left(T_{n}-\lambda I\right)^{-1} e_{n}\right\rangle,
$$

the $(n, n)$-entry in the resolvent $\left(T_{n}-\lambda I\right)^{-1}$. Observe that we here use the notation $e_{0}, \ldots, e_{n}$ for the standard vectors in $\mathbb{R}^{n+1}$, and index rows and columns in the $(n+1) \times(n+1)$ matrix $T_{n}$ from 0 to $n$. Now recall the formula

$$
A^{-1}=\frac{1}{\operatorname{det} A} \operatorname{adj}(A)
$$


for the inverse of a matrix $A$ in terms of the so-called adjoint of $A$ :

$$
\operatorname{adj}(A)=(\operatorname{cof}(A))^{T} \text {. }
$$

Using this gives us the following:

$$
\left\langle e_{n},\left(T_{n}-\lambda I\right)^{-1} e_{n}\right\rangle=\frac{\operatorname{det}\left(T_{n-1}-\lambda I\right)}{\operatorname{det}\left(T_{n}-\lambda I\right)}=-\frac{\pi_{n}(\lambda)}{\pi_{n+1}(\lambda)},
$$

where $\pi$ are the monic orthogonal polynomials. The spectral theorem gives us:

$$
T_{n}=U_{n} \Lambda U_{n}^{T}
$$

where $U_{n}$ is the orthogonal matrix whose columns are the eigenvectors of $T_{n}$ and $\Lambda=\left(\lambda_{0}, \ldots, \lambda_{n}\right)$ is the diagonal matrix of eigenvalues. Write $U_{n}=\left(f_{j}(i)\right)$. Using the spectral theorem

$$
T_{n}-\lambda I=U_{n} \Lambda U_{n}^{T}-\lambda I=U_{n}(\Lambda-\lambda I) U_{n}^{T},
$$

so that

$$
\left(T_{n}-\lambda I\right)^{-1}=U_{n}(\Lambda-\lambda I)^{-1} U_{n}^{T}
$$

From equation (4.25) we get

$$
\begin{aligned}
-\frac{\pi_{n}(\lambda)}{\pi_{n+1}(\lambda)} & =\left\langle e_{n},\left(T_{n}-\lambda I\right)^{-1} e_{n}\right\rangle=\left\langle e_{n}, U_{n}(\Lambda-\lambda I)^{-1} U_{n}^{T} e_{n}\right\rangle \\
& =\left\langle U_{n}^{T} e_{n},(\Lambda-\lambda I)^{-1} U_{n}^{T} e_{n}\right\rangle=\sum_{j=0}^{n} \frac{f_{j}^{2}(n)}{\lambda_{j}-\lambda} .
\end{aligned}
$$

Therefore,

$$
\sum_{j=0}^{n} \frac{f_{j}^{2}(n)}{\lambda-\lambda_{j}}=\frac{\pi_{n}(\lambda)}{\pi_{n+1}(\lambda)} .
$$

By looking at the left-hand side of the equation, we realise that when $\lambda$ is close to $\lambda_{j}$ the ratio will give us either big, positive values or big negative values, depending on whether $\lambda$ gets closer to $\lambda_{j}$ from the left-hand side or the right-hand side. The derivative

$$
\frac{d}{d \lambda} \sum f_{j}^{2}(n) /\left(\lambda-\lambda_{j}\right)=-\sum f_{j}^{2}(n) /\left(\lambda-\lambda_{j}\right)^{2}
$$

is negative, so that the function in the left-hand side of equation (4.28) is decreasing everywhere.

The graph of the function in the left-hand side of equation (4.28) can be visualized as in Figure 1 below.

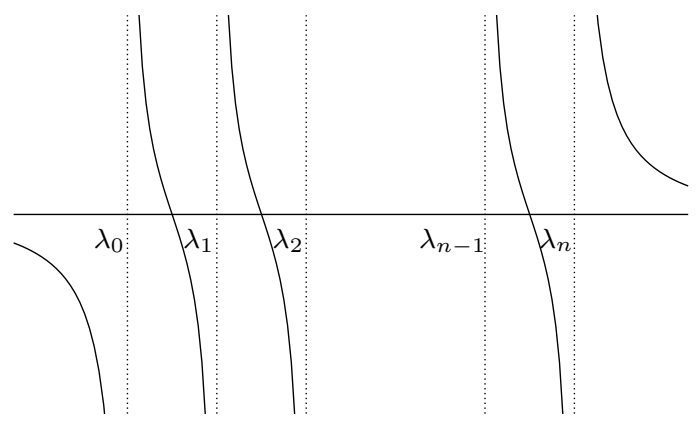

Figure 1: Interlacing zeros of orthogonal polynomials. 
Thus, the left-hand side of equation (4.28) must have a zero between any two zeros of $\pi_{n+1}(\lambda)$. But this must be a zero of $\pi_{n}(\lambda)$.

For further theory about orthogonal polynomials, see [1].

\section{Classical orthogonal polynomials}

Many orthogonal polynomials are named after the ones who discovered them. This thesis will focus on three of these orthogonal polynomials.

- Legendre polynomials

- Hermite polynomials

- Laguerre polynomials

The polynomials mentioned above are sometimes called classical orthogonal polynomials.

They are polynomials orthogonal with respect to different weight functions. It turns out that they are also solutions to differential equations.

\subsection{Legendre polynomials}

Consider the polynomials which are orthogonal on the interval $[-1,1]$ with respect to the inner product

$$
\langle f, g\rangle=\int_{-1}^{1} f(x) g(x) d x
$$

i.e. w.r.t. the weight function $\omega(x)=1$. The Legendre polynomials $P_{n}(x)$ are defined by

$$
p_{n}(x)=\sqrt{\frac{2 n+1}{2}} P_{n}(x)
$$

where $p_{n}(x)$ denotes the orthonormal polynomial. It turns out that the Legendre polynomials are given by the formula

$$
P_{n}(x)=\frac{1}{2^{n} n !} \frac{d^{n}}{d x^{n}}\left[\left(x^{2}-1\right)^{n}\right]
$$

Equation (5.2) is called Rodrigues' formula. Using the binomial theorem on $\left(x^{2}-1\right)^{n}$ and differentiating the result $n$ times, it follows that:

$$
P_{n}(x)=\sum_{j=0}^{N}(-1)^{j} \frac{(2 n-2 j) !}{2^{n} j !(n-j) !(n-2 j) !} x^{n-2 j}
$$

where $N=n / 2$ if $n$ is even and $N=(n-1) / 2$ if $n$ is odd. The six first Legendre polynomials will, by help of equation (5.3), therefore be:

$$
\begin{aligned}
& \begin{array}{l}
P_{0}(x)=1 \quad P_{1}(x)=x
\end{array} \\
& P_{2}(x)=\frac{1}{2}\left(3 x^{2}-1\right) \quad P_{3}(x)=\frac{1}{2}\left(5 x^{3}-3 x\right) \\
& P_{4}(x)=\frac{1}{8}\left(35 x^{2}-30 x^{2}+3\right) \quad P_{5}(x)=\frac{1}{8}\left(63 x^{5}-70 x^{3}+15 x\right)
\end{aligned}
$$


Theorem 4. The polynomials $p_{n}(x)$ defined from equations (5.1) and (5.2) are orthonormal i.e. the Legendre polynomials satisfy

$$
\left\langle P_{n}, P_{m}\right\rangle= \begin{cases}0 & \text { if } m \neq n, \\ \frac{2}{2 n+1} & \text { if } m=n .\end{cases}
$$

Proof. For $m=n$, we must show that

$$
\left\|P_{n}\right\|=\left[\int_{-1}^{1} P_{n}^{2}(x) d x\right]^{1 / 2}=\sqrt{\frac{2}{2 n+1}} .
$$

Let $u=x^{2}-1$. The function $u^{n}$ and its derivatives $\left(u^{n}\right)^{\prime}, \ldots,\left(u^{n}\right)^{(n-1)}$ are zero at $x= \pm 1$, and $\left(u^{n}\right)^{(2 n)}=(2 n)$ !. Integrating by parts $n$ times, using equation (5.2) we get:

$$
\begin{aligned}
\left(2^{n} n !\right)^{2}\left\|P_{n}\right\|^{2} & =\int_{-1}^{1}\left(u^{n}\right)^{(n)}\left(u^{n}\right)^{(n)} d x \\
& =\left.\left(u^{n}\right)^{(n-1)}\left(u^{n}\right)^{(n)}\right|_{-1} ^{1}-\int_{-1}^{1}\left(u^{n}\right)^{(n-1)}\left(u^{n}\right)^{(n+1)} d x \\
& =\cdots \\
& =(-1)^{n}(2 n) ! \int_{-1}^{1} u^{n} d x \\
& =2(2 n) ! \int_{0}^{1}\left(1-x^{2}\right)^{n} d x .
\end{aligned}
$$

Doing the substitution $x=\sin \tau$ and we get:

$$
\int_{0}^{1}\left(1-x^{2}\right)^{n} d x=\int_{0}^{\pi / 2} \cos ^{2 n+1} \tau d \tau=\int_{0}^{\pi / 2} \cos \tau \cdot \cos ^{2 n} \tau d \tau
$$

Let $I_{n}=\int_{0}^{\pi / 2} \cos \tau \cdot \cos ^{2 n} \tau d \tau$. Clearly $I_{0}=1$. Doing integration by parts, we get:

$$
\begin{aligned}
I_{n}=\left.\left(\sin \tau \cdot \cos ^{2 n} \tau\right)\right|_{0} ^{\pi / 2}-\int_{0}^{\pi / 2} \sin \tau \cdot 2 n \cos ^{2 n-1} \tau(-\sin \tau) d \tau= \\
=2 n \int_{0}^{\pi / 2} \cos ^{2 n-1} \tau\left(1-\cos ^{2} \tau\right) d \tau \\
=2 n \int_{0}^{\pi / 2} \cos ^{2 n-1} \tau-\cos ^{2 n+1} d \tau \\
=2 n\left(I_{n-1}-I_{n}\right) .
\end{aligned}
$$

That is,

$$
I_{n}=2 n\left(I_{n-1}-I_{n}\right)
$$

which gives

$$
I_{n}=\frac{2 n}{2 n+1} I_{n-1}
$$


Repeated use of equation (5.5) gives

$$
I_{n}=\frac{2 n}{2 n+1} \cdot \frac{2(n-1)}{2(n-1)+1} I_{n-2}=\cdots=\frac{2 n \cdot 2(n-1) \cdot \ldots \cdot 2}{(2 n+1)(2 n-1) \cdot \ldots \cdot 3} I_{0} .
$$

Extend both numerator and denominator with the even factors $2 n,(2 n-2)$, $(2 n-4), \ldots, 2$, it follows that

$$
\left(2^{n} n !\right)^{2}\left\|P_{n}\right\|^{2}=2(2 n) ! \cdot \frac{2^{2 n} n^{2}(n-1)^{2}(n-2)^{2} \cdot \ldots \cdot 1^{2}}{(2 n+1)(2 n) !}=\frac{2^{2 n+1}(n !)^{2}}{(2 n+1)(2 n) !} .
$$

Now dividing both sides with $\left(2^{n} n !\right)^{2}$ gives that

$$
\left\|P_{n}\right\|^{2}=\frac{2}{2 n+1}
$$

To complete the proof it is enough to check that

$$
\left\langle x^{m}, P_{n}\right\rangle=0
$$

for $m<n$. But for $m<n$,

$$
\begin{aligned}
2^{n} n !\left\langle x^{m}, P_{n}\right\rangle & =\int_{-1}^{1} x^{m}\left(u^{n}\right)^{(n)} d x \\
& =\left.x^{m}\left(u^{n}\right)^{(n-1)}\right|_{-1} ^{1}-m \int_{-1}^{1} x^{m-1}\left(u^{n}\right)^{(n-1)} d x \\
& =\cdots \\
& =(-1)^{m} m ! \int_{-1}^{1}\left(u^{n}\right)^{(n-m)} d x \\
& =\left.(-1)^{m} m !\left(u^{n}\right)^{(n-m-1)}\right|_{-1} ^{1}=0
\end{aligned}
$$

since $n-m-1$ is an integer $k$ such that $0 \leq k \leq n-1$.

The Legendre polynomials are known to be solutions of the Legendre differential equation (5.6)

$$
\left(1-x^{2}\right) P_{n}^{\prime \prime}-2 x P_{n}^{\prime}+n(n+1) P_{n}=0
$$

\subsection{Hermite polynomials}

The second class of polynomials are the Hermite polynomials. They are orthogonal on $\mathbb{R}$ with respect to the inner product

$$
\langle f, g\rangle=\int_{-\infty}^{\infty} f(x) g(x) e^{-x^{2}} d x
$$

i.e. with the weight function $\omega(x)=e^{-x^{2}}$. 
The Hermite polynomials $H_{n}(x)$ are defined by

$$
p_{n}(x)=\frac{1}{\left(2^{n} n ! \sqrt{\pi}\right)^{1 / 2}} H_{n}(x),
$$

where $p_{n}(x)$ is the sequence of orthonormal polynomials. It can be shown that

$$
H_{n}(x)=(-1)^{n} e^{x^{2}} \frac{d^{n}}{d x^{n}}\left(e^{-x^{2}}\right) .
$$

When developing the differentials from equation (5.10) we get:

$$
H_{n}(x)=n ! \sum_{j=0}^{N}(-1)^{j} \frac{2^{n-2 j}}{j !(n-2 j) !} x^{n-2 j}
$$

where $N=n / 2$ if $n$ is even and $N=(n-1) / 2$ if $n$ is odd. With help from equation (5.10) the six first polynomials can be computed:

$$
\begin{aligned}
& H_{0}(x)=1 \quad H_{1}(x)=2 x \\
& H_{2}(x)=4 x^{2}-2 \quad H_{3}(x)=8 x^{3}-12 x \\
& H_{4}(x)=16 x^{4}-48 x^{2}+12 \quad H_{5}(x)=32 x^{5}-160 x^{3}+120 x
\end{aligned}
$$

Theorem 5. The polynomials $p_{n}(x)$ defined by equation (5.8) and (5.9) are orthonormal, i.e. the Hermite polynomials satisfy

$$
\int_{-\infty}^{\infty} e^{-x^{2}} H_{m}(x) H_{n}(x) d x= \begin{cases}0 & \text { if } m \neq n \\ 2^{n} n ! \sqrt{\pi} & \text { if } m=n .\end{cases}
$$

Proof. Equation (5.10) can also be written as:

$$
H_{n}(x)=\sum_{j=0}^{N} \frac{(-1)^{j}}{j !} n(n-1) \cdots(n-2 j+1)(2 t)^{n-2 j}
$$

when $n=2,3,4 \ldots$. For $n \geq 1$ we get following from differentiating:

$$
H_{n}^{\prime}(x)=2 n \sum_{j=0}^{M} \frac{(-1)^{j}}{j !}(n-1)(n-2) \cdots(n-2 j)(2 t)^{n-1-2 j}=2 n H_{n-1}(x)
$$

where $M=(n-2) / 2$ if $n$ is even and $M=(n-1) / 2$ if $n$ is odd. To prove the theorem we apply equation (5.12) to $H_{m}$, and we assume that $m \leq n$. Note that, since $\omega(x)=e^{-x^{2}}$ it follows from equation (5.9) that

$$
(-1)^{n} e^{-x^{2}} H_{n}(x)=\omega^{(n)}(x) .
$$


Integrating by parts, $m$ times, we get

$$
\begin{aligned}
(-1)^{n} \int_{-\infty}^{\infty} e^{-x^{2}} H_{m}(x) H_{n}(x) d x= & \int_{-\infty}^{\infty} H_{m}(x) \omega^{(n)}(x) d x \\
& =\left.H_{m}(x) \omega^{(n-1)}(x)\right|_{-\infty} ^{\infty}-\int_{-\infty}^{\infty} 2 m H_{m-1} \omega^{(n-1)}(x) d x \\
& =-2 m \int_{-\infty}^{\infty} H_{m-1}(x) \omega^{(n-1)}(x) d x \\
& =\cdots \\
& =(-1)^{m} 2^{m} m ! \int_{-\infty}^{\infty} H_{0}(x) \omega^{(n-m)}(x) d x .
\end{aligned}
$$

In the last row of the equation, $H_{0}(x)=1$. If $m<n$, integrating once more, we obtain 0 since $\omega$ and its derivatives approach 0 as $x \rightarrow \pm \infty$. If $m=n$, the integral in the last row equals:

$$
\int_{-\infty}^{\infty} e^{-x^{2}} d x=\sqrt{\pi}
$$

There is a differential equation that the Hermite polynomials satisfy, called the Hermite differential equation:

$$
H_{n}^{\prime \prime}-2 x H_{n}^{\prime}+2 n H_{n}=0
$$

\subsection{Laguerre polynomials}

Consider the polynomials which are orthogonal on $[0, \infty)$ with respect to the inner product

$$
\langle f, g\rangle=\int_{0}^{\infty} f(x) g(x) e^{-x} d x
$$

i.e. the weight function $\omega(x)=e^{-x}$. The Laguerre polynomials are defined by

$$
p_{n}(x)=e^{-x / 2} L_{n}(x)
$$

where $p_{n}(x)$ is the sequence of orthonormal polynomials. It can be shown that

$$
L_{n}(x)=\frac{e^{x}}{n !} \frac{d^{n}}{d x^{n}}\left(x^{n} e^{-x}\right), \quad n=0,1,2, \ldots
$$

Equation (5.16) can be expressed without the differentiation. Then it will look like

$$
L_{n}(x)=\sum_{j=0}^{n} \frac{(-1)^{j}}{j !}\left(\begin{array}{l}
n \\
j
\end{array}\right) x^{j} .
$$

Using the formula from equation (5.17), the first five polynomials can be calculated:

$$
\begin{array}{ll}
L_{0}(x)=1 & L_{1}(x)=-x+1 \\
L_{2}(x)=\frac{1}{2}\left(x^{2}-4 x+2\right) & L_{3}(x)=\frac{1}{6}\left(-x^{3}+9 x^{2}-18 x+6\right) \\
L_{4}(x)=\frac{1}{24}\left(x^{4}-16 x^{3}+72 x^{2}-96 x+24\right) &
\end{array}
$$


The Laguerre polynomials are a solution to a differential equation, called the Laguerre differential equation:

$$
x L_{n}^{\prime \prime}+(1-x) L_{n}^{\prime}+n L_{n}=0 .
$$

The above results concerning classical orthogonal polynomials can be found in [3].

\section{Sturm-Liouville problems}

Many mathematical and physical problems lead to a differential equation of the form:

$$
\left(p(x) v^{\prime}\right)^{\prime}+q(x) v+\lambda r(x) v=0 \quad a<x<b .
$$

In addition one has boundary conditions at the end points of the interval. The differential equation (6.1) together with boundary conditions is called a Sturm-Liouville eigenvalue problem, and a solution is called an eigenfunction with eigenvalue $\lambda$.

Example 7. Consider the Sturm-Liouville problem

$$
\begin{gathered}
\frac{d^{2} v}{d x^{2}}+\lambda v=0 \quad 0<x<L, \\
v(0)=v(L)=0,
\end{gathered}
$$

where $p=r=1$ and $q=0$. This boundary condition is called a Dirichlet boundary condition. The eigenfunctions and eigenvalues for this problem are

$$
v_{n}(x)=\sin \frac{n \pi x}{L}, \quad \lambda_{n}=\left(\frac{n \pi}{L}\right)^{2} \quad n=1,2,3, \ldots
$$

Example 8. Consider the Sturm-Liouville problem

$$
\begin{gathered}
\frac{d^{2} v}{d x^{2}}+\lambda v=0 \quad 0<x<L, \\
v^{\prime}(0)=v^{\prime}(L)=0,
\end{gathered}
$$

where $p=r=1$ and $q=0$. This boundary conditions is called a Neumann boundary condition. The eigenfunctions and eigenvalues for this problem are

$$
v_{n}(x)=\cos \frac{n \pi x}{L}, \quad \lambda_{n}=\left(\frac{n \pi}{L}\right)^{2} \quad n=0,1,2, \ldots
$$

Example 9. Consider the Sturm-Liouville problem with

$$
\begin{gathered}
\frac{d^{2} v}{d x^{2}}+\lambda v=0 \quad 0<x<L, \\
v(0)=v(L), \quad v^{\prime}(0)=v^{\prime}(L),
\end{gathered}
$$

where $p=r=1$ and $q=0$. This kind of boundary condition is called a periodic boundary condition. The eigenfunctions and eigenvalues for this problem are

$$
v_{n}(x)=\alpha_{n} \cos \left(\frac{2 n \pi x}{L}\right)+\beta_{n} \sin \left(\frac{2 n \pi x}{L}\right), \quad \lambda_{n}=\left(\frac{2 n \pi}{L}\right)^{2} \quad n=1,2,3, \ldots .
$$

The results above can all be shown by elementary computations using the fact that the eigenvalues must be real. 


\section{Example 10. The Bessel equation.}

For $\nu \in[0, \infty)$, consider the differential equation

$$
r^{2} w^{\prime \prime}(r)+r w^{\prime}(r)+\left(r^{2}-\nu^{2}\right) w(r)=0, \quad r>0 .
$$

The equation above is called the Bessel equation of order $\nu$.

Divide (6.8) with $r$ and put $v(x)=w(r)$ where $x=r / \sqrt{\lambda}$. Then

$$
\left(x v^{\prime}(x)\right)^{\prime}+\left(\lambda x-\frac{\nu^{2}}{x}\right) \nu(x)=0 .
$$

This equation has the form of a the Sturm-Liouville equation with $p(x)=r(x)=x$ and $q(x)=-\nu^{2} / x$. The bounded solution of equation (6.8), denoted $J_{\nu}$, is called the Bessel function (of the first kind) of order $\nu$.

Theorem 6. Consider the Sturm-Liouville equation

$$
\left(p(x) v^{\prime}\right)^{\prime}+q(x) v+\lambda r(x) v=0, \quad a<x<b,
$$

together with Dirichlet, Neumann or periodic boundary conditions. Then the eigenfunctions corresponding to different eigenvalues are orthogonal with respect to a inner product

$$
\langle u, v\rangle_{r}=\int_{a}^{b} u(x) v(x) r(x) d x
$$

Proof. Let us write

$$
L v:=\left(p v^{\prime}\right)^{\prime}+q v
$$

Using the product rule, we get:

$$
\begin{aligned}
u L v-v L u & =u\left(p v^{\prime}\right)^{\prime}+u q v-v\left(p u^{\prime}\right)^{\prime}-v q u \\
& =\left(u p v^{\prime}\right)^{\prime}-u^{\prime} p v^{\prime}-\left(v p u^{\prime}\right)^{\prime}+u^{\prime} p v^{\prime} .
\end{aligned}
$$

Equation (6.12) can be simplified to

$$
u L v-v L u=\left[p\left(u v^{\prime}-v u^{\prime}\right)^{\prime}\right]^{\prime}
$$

Equation (6.13) is called the Lagrange identity. Integrating equation (6.13) over the interval $[a, b]$, we get following equation, which is called Green's formula:

$$
\int_{a}^{b}(u L v-v L u) d x=\left.p\left(u v^{\prime}-v u^{\prime}\right)\right|_{a} ^{b}
$$

Assuming that $u$ and $v$ satisfy the boundary conditions, then

$$
\left.p\left(u v^{\prime}-v u^{\prime}\right)\right|_{a} ^{b}=0
$$

which leads to

$$
\int_{a}^{b}(u L v-v L u) d x=0
$$


Now assume that $v_{n}$ and $v_{m}$ are two eigenfunctions with two different eigenvalues, $\lambda_{n}$ and $\lambda_{m}$. Then

$$
\begin{aligned}
& -L v_{n}=\lambda_{n} r v_{n}, \\
& -L v_{m}=\lambda_{m} r v_{m} .
\end{aligned}
$$

If we multiply equation (6.17) with $v_{m}$ and equation (6.18) with $v_{n}$, integrating them over the interval $[a, b]$ and finally taking the difference of these two equations, we obtain the following expression:

$$
-\int_{a}^{b}\left(v_{m} L v_{n}-v_{n} L v_{m}\right) d x=\left(\lambda_{n}-\lambda_{m}\right) \int_{a}^{b} v_{n} v_{m} r d x
$$

Applying Green's formula we get that

$$
\left(\lambda_{n}-\lambda_{m}\right) \int_{a}^{b} v_{n} v_{m} r d x=0 .
$$

Since $\lambda_{n} \neq \lambda_{m}$ it follows that

$$
\left\langle v_{n}, v_{m}\right\rangle_{r}=\int_{a}^{b} v_{n} v_{m} r d x=0
$$

proving orthogonality.

For further theory about Sturm-Liouvlle problems, see [4, Pinchover].

The three classical orthogonal polynomials were all solutions to differential equations:

- The Legendre differential equation

$$
\left(1-x^{2}\right) v^{\prime \prime}-2 x v^{\prime}+\lambda v=0
$$

- The Hermite differential equation

$$
v^{\prime \prime}-2 x v^{\prime}+\lambda v=0
$$

- The Laguerre differential equation

$$
x v^{\prime \prime}+(1-x) v^{\prime}+\lambda v=0
$$

They can all be written in the form of Sturm-Liouville equation for different choices of $p, q$ and $r$.

The Legendre differential equation can, clearly, be written in the form (6.1), where $p=\left(1-x^{2}\right), q=0$ and $r=1$. Here $[a, b]=[-1,1]$.

Now consider the Hermite differential equation. After multiplying both sides of equation (6.1) with $e^{-x^{2}}$ we see that

$$
e^{-x^{2}} v^{\prime \prime}-2 x e^{-x^{2}}+\lambda e^{-x^{2}} v=0
$$

or

$$
\left(e^{-x^{2}} v^{\prime}\right)^{\prime}+\lambda e^{-x^{2}} v=0
$$


This gives us: $p=e^{-x^{2}}, q=0$ and $r=e^{-x^{2}}$. Here $(a, b)=(-\infty, \infty)$.

Finally consider the Laguerre differential equation. Multiplying both sides of (6.1) with $e^{-x}$, we find

$$
e^{-x} x v^{\prime \prime}+e^{-x}(1-x) v^{\prime}+e^{-x} \lambda v=0 .
$$

It is easy to see that this can be rewritten as

$$
\left(e^{-x} x v^{\prime}\right)^{\prime}+e^{-x} \lambda v=0,
$$

that is $p=x e^{-x}, q=0$ and $r=e^{-x}$. Here $[a, b)=[0, \infty)$.

It is interesting to note that the orthogonality of the classical orthogonal polynomials with respect to the weights indicated in the previous work can also be seen from the proof of Theorem 6. Indeed formula (6.15) still holds true, even in the absence of boundary conditions. Simply note that $p$ vanishes at the ends of the intervals (and, in case the intervals are unbounded, $p$ decays faster than the polynomials).

For more about the theory of Sturm-Liouville problems, see [4].

\section{Applications}

\subsection{Eigenvalues of the Laplace operator}

Let $\Omega$ be a bounded open subset of $\mathbb{R}^{n}$. The eigenvalue problem for the Dirichlet Laplace operator

$$
\Delta=\frac{\partial^{2}}{\partial x_{1}^{2}}+\cdots+\frac{\partial^{2}}{\partial x_{n}^{2}}
$$

is then given by the equation

$$
-\Delta u=\lambda u,
$$

where $u$ is a function in $\Omega$ such that $u=0$ on $\partial \Omega$.

One can show that the Dirichlet Laplace operator has discrete eigenvalues $\left(\lambda_{j}\right)_{1}^{\infty}$,

$$
0<\lambda_{1} \leq \lambda_{2} \leq \lambda_{3} \leq \ldots,
$$

accumulating at infinity. They are important in many applications. For example, consider the wave equation

$$
\frac{\partial^{2} v}{\partial t^{2}}-c^{2} \Delta v=0
$$

for the amplitude $v(x, t)$ of a wave at the position $x=\left(x_{1}, \ldots, x_{n}\right)$ at time $t$. Separating variables by writing $v(x, t)=u(x) T(t)$ we find that

$$
u T^{\prime \prime}-c^{2} \Delta u T=0 .
$$

Dividing both sides by $c^{2} u T$, it follows that

$$
\frac{\Delta u}{u}=\frac{T^{\prime \prime}}{c^{2}} .
$$

Both sides must be constant, i.e. independent of $(x, t)$, and denoting the constant by $-\lambda$ we find that $u$ must satisfy the equation (7.1), whereas

$$
T^{\prime \prime}+\lambda c^{2} T=0 .
$$


The solutions of equation (7.2) describe periodic motion depending on the frequency $\omega$, where $\omega^{2}=\lambda c^{2}$. Note that the frequencies are determined by the eigenvalues of the Laplacian.

\subsubsection{Eigenvalue problem in a disk}

To find the frequencies generated by a circular drum we must find the eigenvalues for the Dirichlet Laplace operator in a disk. Let $\Omega$ be the disk defined by

$$
\{0 \leq r<a, 0 \leq \theta \leq 2 \pi\}
$$

The eigenvalue problem (7.1), written in polar coordinates, becomes

$$
u_{r r}+\frac{1}{r} u_{r}+\frac{1}{r^{2}} u_{\theta \theta}=-\lambda u
$$

where $0<r<a, 0 \leq \theta \leq 2 \pi$ and $u(a, \theta)=0$. To find the solutions we separate variables: $u(r, \theta)=R(r) \Theta(\theta)$. Then we get two Sturm-Liouville problems:

$$
\Theta^{\prime \prime}(\theta)+\mu \Theta(\theta)=0
$$

where $\Theta(0)=\Theta(2 \pi)$, and

$$
R^{\prime \prime}(r)+\frac{1}{r} R^{\prime}(r)+\left(\lambda-\frac{\mu}{r^{2}}\right) R(r)=0 .
$$

The solutions of the first problem is

$$
\Theta_{n}(\theta)=A_{n} \cos n \theta+B_{n} \sin n \theta
$$

where $\mu_{n}=n^{2}$ for $n=1,2,3, \ldots$ Using this, the second Sturm-Liouville problem (equation (7.5)) can be written as:

$$
R^{\prime \prime}(r)+\frac{1}{r} R^{\prime}(r)+\left(\lambda-\frac{n^{2}}{r^{2}}\right) R(r)=0 .
$$

Doing the change of variables $s=\sqrt{\lambda} r$ and $R(r)=\Psi(s)$ we get:

$$
R^{\prime}(r)=\frac{d}{d r} R(r)=\frac{d}{d r} \Psi(\sqrt{\lambda} r)=\Psi^{\prime}(\sqrt{\lambda} r) \cdot \sqrt{\lambda}
$$

and

$$
R^{\prime \prime}(r)=\Psi^{\prime \prime}(s) \cdot \lambda
$$

Therefore equation (7.7)

$$
\lambda \Psi^{\prime \prime}(s)+\frac{\sqrt{\lambda}}{s} \Psi^{\prime}(s) \sqrt{\lambda}+\left(\lambda-\frac{n^{2} \lambda}{s^{2}}\right) \Psi(s)=0 .
$$

Dividing both sides with $\lambda$, it follows

$$
\Psi^{\prime \prime}(s)+\frac{1}{s} \Psi^{\prime}(s)+\left(1-\frac{n^{2}}{s^{2}}\right) \Psi(s)=0 .
$$


After multiplying both sides of equation (7.11) by $s^{2}$, we see that $\Psi$ satisfies the Bessel equation of order $n$, compare (6.8). Since $\Psi$ is bounded, we have that $\Psi(s)=J_{n}(s)$. The function $R(r)=J_{n}(s)=J_{n}(\sqrt{\lambda} r)$ must vanish when $r=a$. If we let $\left(\alpha_{n, m}\right)_{m=1}^{\infty}$ denote the zeros of the function $J_{n}$ on the positive real line, we see that the eigenvalues $\lambda$ are given by

$$
\sqrt{\lambda} a=\alpha_{n, m}
$$

or

$$
\lambda=\frac{\left(\alpha_{n, m}\right)^{2}}{a^{2}} .
$$

This implies that the frequencies that a circular drum can generate are given by

$$
\omega=\sqrt{\lambda} c=\frac{\alpha_{n, m}}{a} c .
$$

\subsubsection{Eigenvalue problem in a ball}

Imagine a solid ball of radius $a$ given in spherical coordinates. Let us introduce the spherical coordinates

$$
\begin{aligned}
& x=r \sin \phi \cos \theta, \\
& y=r \sin \phi \sin \theta, \\
& z=r \cos \phi,
\end{aligned}
$$

see Figure 2 below.

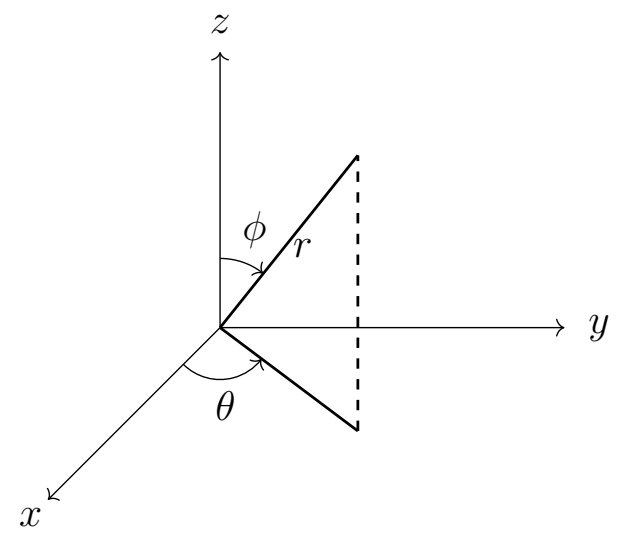

Figure 2: Notation for a spherical coordinate system.

We define $B_{a}$ and $S^{2}$ by

$B_{a}=\{0 \leq r<a, 0 \leq \phi \leq \pi, 0 \leq \theta \leq 2 \pi\}$ and $S^{2}=\{0 \leq \phi \leq \pi, 0 \leq \theta \leq 2 \pi\}$.

The eigenvalue problem for the Dirichlet Laplace operator in $B_{a}$, written in spherical coordinates, becomes

$$
\begin{aligned}
\frac{1}{r^{2}} \frac{\partial}{\partial r}\left(r^{2} \frac{\partial u}{\partial r}\right) & +\frac{1}{r^{2}}\left[\frac{1}{\sin \phi} \frac{\partial}{\partial \phi}\left(\sin \phi \frac{\partial u}{\partial \phi}\right)+\frac{1}{\sin ^{2} \phi} \frac{\partial^{2} u}{\partial \theta^{2}}\right] \\
& =-\lambda u, \quad(r, \phi, \theta) \in B_{a}, \\
u & (a, \phi, \theta)=0, \quad(\phi, \theta) \in S^{2} .
\end{aligned}
$$


The function $u(r, \phi, \theta)$ can be expressed, using the method of separating variables, as

$$
u(r, \phi, \theta)=R(r) Y(\phi, \theta) .
$$

We obtain two eigenvalue problems, one over the unit sphere $S^{2}$ and the one for the radial function $R(r)$. The eigenvalue problem over the unit sphere takes the form

$$
\frac{1}{\sin \phi} \frac{\partial}{\partial \phi}\left(\sin \phi \frac{\partial Y}{\partial \phi}\right)+\frac{1}{\sin ^{2} \phi} \frac{\partial^{2} Y}{\partial \theta^{2}}=-\mu Y \quad(\phi, \theta) \in S^{2}
$$

with the boundary condition

$$
Y(\phi, 0)=Y(\phi, 2 \pi), \quad Y_{\theta}(\phi, 0)=Y_{\theta}(\phi, 2 \pi) .
$$

The second eigenvalue problem takes the form

$$
\frac{1}{r^{2}} \frac{d}{d r}\left(r^{2} \frac{d R}{d r}\right)=\left(\frac{\mu}{r^{2}}-\lambda\right) R \quad 0<r<a,
$$

with boundary condition $R(a)=0$. The function $Y$ can be expressed, using the method of separating variables, as

$$
Y(\phi, \theta)=\Phi(\phi) \Theta(\theta) .
$$

Substituting this form of $Y$ into equation (7.14), we obtain

$$
\Theta^{\prime \prime}(\theta)+\nu \Theta(\theta)=0
$$

where $0<\theta<2 \pi$, and

$$
\sin \phi \frac{d}{d \phi}\left[\sin \phi \frac{d \Phi(\phi)}{d \phi}\right]+\left(\mu \sin ^{2} \phi-\nu\right) \Phi(\phi)=0,
$$

where $0<\phi<\pi$. The periodic boundary condition from equation (7.15) gives us the eigenvalues $\nu_{m}=m^{2}$ and eigenfunctions

$$
\Theta_{m}(\theta)=A_{m} \cos m \theta+B_{m} \sin m \theta
$$

for $m=0,1,2, \ldots$. Substituting $\nu=m^{2}$ into the equation (7.18) for $\Phi(\phi)$ and doing the change of variables $x=\cos \phi, P(x)=\Phi(\phi(x))$, we get

$$
\left(1-x^{2}\right) \frac{d}{d x}\left[\left(1-x^{2}\right) \frac{d P}{d x}\right]+\left[\left(1-x^{2}\right) \mu-m^{2}\right] P=0
$$

where $-1<x<1$ and $m=0,1,2, \ldots$ For $m=0$ we obtain

$$
\frac{d}{d x}\left[\left(1-x^{2}\right) \frac{d P}{d x}\right]+\mu P=0, \quad-1<x<1,
$$

i.e. the Legendre equation. It turns out that equation (7.20) has bounded solutions if and only if $\mu=n(n+1)$ where $n=0,1,2, \ldots$; they are the Legendre polynomials, $P_{n}$.

For $m>0$, equation (7.19) is called the associated Legendre equation of order $m$. This equation has got bounded solutions when $\mu=n(n+1)$ given by

$$
P_{n}^{m}(x)=\left(1-x^{2}\right)^{m / 2} \frac{d^{m} P_{n}}{d x^{m}}
$$


Note that $P_{n}^{m}=0$ if $m>n$, so we may assume $0 \leq m \leq n$. For equation (7.16) we may substitute $\mu=n(n+1)$ and $R(r)=\rho(r) / \sqrt{r}$, then (7.16) becomes the Bessel equation of order $n+\frac{1}{2}$ :

$$
\rho_{n}^{\prime \prime}(r)+\frac{1}{r} \rho_{n}^{\prime}(r)+\left[\lambda-\frac{\left(n+\frac{1}{2}\right)^{2}}{r^{2}}\right] \rho_{r}(r)=0 .
$$

When doing the change of variables $s=\sqrt{\lambda} r$ in the previous section, we find that from the boundary condition $R(a)=0$ that the radial solution has the form

$$
R_{n, l}(r)=\frac{J_{n+\frac{1}{2}}\left(\sqrt{\lambda_{n, l}} r\right)}{\sqrt{r}}, \quad \lambda_{n, l}=\left(\frac{\alpha_{n, l}}{a}\right)^{2}, \quad l=1,2, \ldots,
$$

where $\alpha_{n, l}$ denote the zeros of the Bessel function $J_{n+\frac{1}{2}}$.

For the eigenvalue problem in a ball, we get following eigenfunctions

$$
U_{n, m, l}(r, \phi, \theta)=\frac{1}{\sqrt{r}} J_{n+\frac{1}{2}}\left(\frac{\alpha_{n, l} r}{a}\right) Y_{n, m}(\phi, \theta),
$$

for $n=0,1, \ldots, m=0,1, \ldots, n$ and $l=1,2, \ldots$, with the eigenvalues

$$
\lambda_{n, l}=\left(\frac{\alpha_{n, l}}{a}\right)^{2}
$$

for $n=0,1, \ldots$ and $l=1,2, \ldots$. Note that the eigenvalue $\lambda_{n, l}$ of the Dirichlet Laplace operator has multiplicity $2 n+1$. The functions $Y_{n, m}(\phi, \theta)$,

$$
Y_{n, m}(\phi, \theta)=\left\{\cos m \theta P_{n}^{m}(\cos \phi), \sin m \phi P_{n}^{m}(\cos \phi)\right\},
$$

for $n=0,1, \ldots$ and $m=0,1, \ldots, n$, are called spherical harmonics of order $n$.

Finding the eigenvalues for the Laplace operator of a ball is important in many applications in physics, such as electromagnetism and hydrodynamics.

\subsection{Schrödinger equation}

In classical physics, the dynamics of a particle can be described with the help of Newton's second law

$$
\bar{F}=m \bar{a} .
$$

If $\bar{F}$ is conservative

$$
\bar{F}=-\nabla V
$$

where $V$ is the potential. In quantum mechanics, the possible energy levels of a particle are given as eigenvalues of the operator

$$
H=-\frac{\hbar^{2}}{2 m} \Delta+V
$$

The operator $H$ is called Hamilton operator or Schrödinger operator. The eigenvalue problem

$$
H \Psi=E \Psi
$$

is called the Schrödinger equation. One is interested in normalized solutions $\Psi \in L^{2}\left(\mathbb{R}^{n}\right)$. The quantity $|\Psi(x)|^{2}$ has the interpretation of probability density for finding the particle at the point $x$. 


\subsubsection{Harmonic oscillator}

A mass, $m$, is attached to the lower end of a spring whose upper end is fixed. The displacement from the equilibrium is proportional to the relative force:

$$
F=-k x=m \frac{d^{2} x}{d t^{2}}
$$

A solution for (7.27) is

$$
x(t)=A \sin (\omega t)+B \cos (\omega t)
$$

where

$$
\omega=\sqrt{\frac{k}{m}}
$$

is the frequency on oscillation and the potential energy is

$$
V(x)=\frac{k x^{2}}{2} .
$$

To solve the quantum problem, we need to find the solution for the Schrödinger equation for the potential

$$
V(x)=\frac{1}{2} m \omega^{2} x^{2}
$$

The Schrödinger equation will look like:

$$
-\frac{\hbar^{2}}{2 m} \Psi^{\prime \prime}+\frac{1}{2} m \omega^{2} x^{2} \Psi=E \Psi
$$

We introduce two new variables:

$$
s=\sqrt{\frac{m \omega}{\hbar}} x
$$

and

$$
\lambda=\frac{2 E}{\hbar \omega}
$$

and put $\Psi(x)=\tilde{\Psi}(s)$. Using these variables, equation (7.32) will look like

$$
\tilde{\Psi}(s)^{\prime \prime}=\left(s^{2}-\lambda\right) \tilde{\Psi}(s) .
$$

For large values of $s$ (and large values of $x$ ) the $s^{2}$-term dominates over $\lambda$ and equation (7.35) can be approximated by

$$
\tilde{\Psi}(s)^{\prime \prime} \approx s^{2} \tilde{\Psi}(s)
$$

which has the approximate solution

$$
\tilde{\Psi}(s) \approx e^{-s^{2} / 2} .
$$

Therefore we make the ansatz

$$
\tilde{\Psi}(s)=h(s) e^{-s^{2} / 2} .
$$

Differentiating this one, the Schrödinger equation from equation (7.35) will look like

$$
\frac{d^{2} h}{d s^{2}}-2 s \frac{d h}{d s}+(\lambda-1) h=0
$$


If we set

$$
\lambda=2 n+1
$$

equation (7.39) will be identical to the Hermite differential equation. Equation (7.34) gives

$$
E=\hbar \omega\left(n+\frac{1}{2}\right), \quad \text { for } n=0,1,2, \cdots
$$

Generally, $h_{n}(s)$ will be a polynomial of degree $n$ in $s$. Finally, the normalized eigenfunctions for the harmonic oscillator are

$$
\Psi_{n}(x)=\left(\frac{m \omega}{\pi \hbar}\right)^{1 / 4} \frac{1}{\sqrt{2^{n} n !}} H_{n}(s) e^{-s^{2} / 2}
$$

where $H_{n}(s)$ are Hermite polynomials of order $n$.

\subsubsection{Hydrogen atom}

The hydrogen atom consists of one nucleus and one electron. The Coulomb attraction between the nucleus and the electron leads to the Schrödinger equation

$$
-\left(\frac{\hbar^{2}}{2 m} \Delta+\frac{e^{2}}{4 \pi \varepsilon_{0} r}\right) \Psi=E \Psi,
$$

where $\hbar$ is the Planck constant divided by $2 \pi, m$ is the mass of the electron, $r$ is the distance between the nucleus (which is fixed at the origin) and the electron, $e$ is the electric charge and $\varepsilon_{0}$ is the vacuum electric permittivity.

As we shall see, the eigenvalue problem (7.42) has an infinite number of discrete negative eigenvalues, which are possible bound states for the hydrogen atom. Due to radial symmetry we put $\Psi(r, \phi, \theta)=R(r) Y_{l, p}(\phi, \theta)$. By computations similar to those used when we computed the eigenvalues for the Laplacian in a ball, we obtain following equation for $R$ :

$$
\left(r^{2} R^{\prime}\right)^{\prime}+\frac{2 m r^{2}}{\hbar^{2}}\left(E+\frac{e^{2}}{r}\right) R-l(l+1) R=0
$$

where $l=0,1,2, \ldots$ In order for $R$ to decay at $\infty$, it is easy to see that $E$ must be negative. It is useful to scale out the energy $E$ from the problem. To do this, we introduce the new variable $\rho=\alpha r$, where

$$
\alpha=\frac{\sqrt{8 m|E|}}{\hbar} .
$$

Equation (7.43) can now be written as

$$
\frac{1}{\rho^{2}}\left(\rho^{2} R^{\prime}\right)^{\prime}+\left[\frac{\lambda}{\rho}-\frac{1}{4}-\frac{l(l+1)}{\rho^{2}}\right] R=0
$$

where

$$
\lambda=\frac{e^{2} \sqrt{m}}{4 \pi \varepsilon_{0} \hbar \sqrt{2|E|}},
$$


and $R=R_{l}(\rho)$. To be able to normalize $\Psi$, we need to look for solutions that approach zero as $\rho \rightarrow \infty$. When $\rho \rightarrow \infty$, equation (7.45) is approximated by $R^{\prime \prime}-\frac{1}{4} R=0$. Let $R=F_{l}(\rho) e^{-\frac{1}{2} \rho}$. Then $F_{l}$ satisfies the following differential equation

$$
F_{l}^{\prime \prime}+\left(\frac{2}{\rho}-1\right) F_{l}^{\prime}+\left[\frac{\lambda-1}{\rho}-\frac{l(l+1)}{\rho^{2}}\right] F_{l}=0 .
$$

By the Frobenius-Fuchs theory, we seek solutions of the form

$$
F_{l}(\rho)=\rho^{\mu_{l}} \sum_{k=0}^{\infty} a_{k}^{l} \rho^{k} .
$$

The indical equation for $\mu_{l}$ is $\mu_{l}\left(\mu_{l}+1\right)-l(l+1)=0$, and the only solution that makes the wave function bounded near the origin is when $\mu_{l}=l$. Substituting the Frobenius-Fuchs ansatz into equation (7.47), leads to the recursion formula

$$
a_{k+1}^{l}=\frac{k+l+1-\lambda}{(k+1)(k+2 l+2)} a_{k}^{l} .
$$

A detailed analysis shows that the solutions which are power series that do not terminate, will grow at $\infty$ at the rate $e^{\rho}$. Thus, the power series must in fact terminate, and the normalizable solutions are polynomials. That is $\lambda=k+l+1$ for some non-negative integer $k$, i.e. $\lambda$ is a positive integer $n$ (such that $n \geq l+1$ ), and we seek solutions of equation (7.47) of the form $F_{l}(\rho)=\rho^{l} \tilde{L}_{l}(\rho)$, where $\tilde{L}_{l}$ is a polynomial. Inserting this into equation (7.47) leads to the following equation for $\tilde{L}_{l}$ :

$$
\rho \tilde{L}_{l}^{\prime \prime}+[2(l+1)-\rho] \tilde{L}_{l}^{\prime}+(\lambda-l-1) \tilde{L}_{l}=0 .
$$

This equation resembles the Laguerre equation. In fact, simple calculations show that if

$$
\rho L_{q}^{\prime \prime}+(1-\rho) L_{q}^{\prime}+q L_{q}=0
$$

then $L_{q}^{(k)}$ satisfies the equation

$$
\rho\left(L_{q}^{(k)}\right)^{\prime \prime}+(k+1-\rho)\left(L_{q}^{(k)}\right)^{\prime}+(q-k)\left(L_{q}^{(k)}\right)=0 .
$$

So, if $\lambda=n$ is a given positive integer and $l \leq n-1$, it follows that

$$
\tilde{L}_{l}=L_{q}^{(k)}=L_{n+l}^{(2 l+1)}
$$

is a (non-zero) polynomial which solves equation (7.49). This leads to (non-normalized) eigenfunctions $\Psi_{n, l, p}$ with eigenvalues

$$
E_{n}=-\frac{m e^{4}}{32 \pi^{2} \varepsilon_{0}^{2} \hbar^{2}} \frac{1}{n^{2}}
$$

as can be seen from equation (7.46). This is the famous Bohr formula. From equation (7.44) and (7.50) it follows that

$$
\rho=\frac{2 r}{n a}
$$


where

$$
a=\frac{4 \pi \varepsilon_{0} \hbar^{2}}{m e^{2}}
$$

is the so-called Bohr radius. The explicit formula for the (non-normalized) eigenfunctions becomes

$$
\Psi_{n, l, p}=\left(\frac{2 r}{n a}\right)^{l} L_{n+l}^{(2 l+1)}\left(\frac{2 r}{n a}\right) Y_{l, p}(\theta, \phi),
$$

where $n=1,2,3, \ldots, l=0,1, \ldots, n-1$ and $p=0,1,2, \ldots, l$. Note that the multiplicity of the eigenvalue $E_{n}$ is

$$
\sum_{l=0}^{n-1}(2 l+1)=n^{2} .
$$

The formula (7.50) leads to the famous Rydberg formula for the wavelength of photons emitted when an electron initially in an excited state falls to a final lower bound state:

$$
\frac{1}{\lambda}=R\left(\frac{1}{n_{f}^{2}}-\frac{1}{n_{i}^{2}}\right)
$$

The constant $R$ is called Rydberg's constant.

Transitions to the ground state $\left(n_{f}=1\right)$ lie in the ultraviolet and are known to spectroscopists as the Lyman series. Transition to the first excited state $\left(n_{f}=2\right)$ lie in the visible region and constitute the Balmer series. Transition to $n_{f}=3$ lie in the infrared and constitute the Paschen series.

The derivation of Rydberg's formula from theoretical principles was one of the early triumphs of quantum mechanics.

For more about quantum mechanics and the Schrödinger equation, see [2] and [4].

\section{Acknowledgement}

I would like to thank my supervisor, Jörgen Östensson, for his help and for inspiring me during this project. 


\section{References}

[1] Percy Deift. Orthogonal polynomials and random matrices: a Riemann-Hilbert approach. Vol. 3. American Mathematical Soc., 1999.

[2] David J Griffiths and Darrell F Schroeter. Introduction to quantum mechanics. Cambridge University Press, 2018.

[3] Erwin Kreyszig. Introductory functional analysis with applications. Vol. 1. wiley New York, 1978.

[4] Yehuda Pinchover and Jacob Rubinstein. An introduction to partial differential equations. Cambridge university press, 2005. 